\title{
Author Index Vol. 4, 1995
}

\section{Author Index Vol. 4,1995}

Abudara,V. 263 Akamatsu;-H. 14 Almaraz, L. 245 Arlt,U. 331

Ballard, H.J. 168 Bicho,M.P. 78 Bienkiewicz, A. 94 Bisgard, G.E. 292 Bolis,C.L. 86 Brown, G.M. 311

Canciglia,P. 86 Casanova, C. 98 Chabli,A. 98 Chan,J.Y.H. 59 Chan,S.H.H. 59 Chan,Y.S. 133 Chang, J.K. 338 Chen, H.I. 186 Chen, J. 277 Chiu,T.H. 117 Chow,P.H. 212 Chuang, J.-i. 325 Claustrat.B. 32 Coote,J.H. 142 Crespo, M.E. 78 Cutrera,R.A. 51

Daniels, W.M.U. 325 Dieter, P. 331 Dinger, B.G. 109,277 Donnelly, D.F. 304 Du,Q. 232 Dun,NJ. 117 Dun,S.L. 117

Eyzaguirre, C. 263

Fidone,S.J. 109,277 Fitzgerald, R.S. 298 Fitzke,E. 331 Fu, G.Q. 232 Fujiwara.N. 195 Geoffriau,M. 32 Gonzalez, C. 245 Greger, J. 72

Han,C. 150 Ho.S.Y. 338 Huang, R. 117

Ide,T. 298 Iizawa, O. 14

Kao,K.-M. 124 Kirsch,R. 42 Kou.Y.R. 271 Kravtsov, G.M. 160 Kumar, G.K. 271 Kuo.T.BJ. 59 Kwan,C.Y. 160 
Lachowicz, A. 206 Lahiri.S. 257 Lee, P.P.N. 212 Lewinski, A. 72 Li,J.C. 225 Li, P. 124 Li,Q.H. 338 Li,Y.L 338 Lin,Q. 124 Liu,K. 232 Liu,YX. 232 Lu,Z. 150 Lung,M.A. 179 Lydic,R. 298

Ma,K.C. 225 Magistretti, P.J. 86 Martin, J.-L. 86 Mauviard, F. 32 Melchiorri, D. 325 Michaud.Y. 98 Modrzejewska, H. 72 Molotchnikoff, S. 98

Niwa, Y 14 Nurse, C.A. 286

Ochedalski,T. 206 Ortiz, G.G. 325 Ouarour,A. 42,51

Pablos,M.L 325 Pang,C.S. 311 Pang,S.F. 24,212,311 Pawlikowski, M. 206 P<§vet,P. 32,42,51 Poeggeler, B. 325 Prabhakar,N.R. 271 Pun,K.K. 19

Qu,P. 150

Randall, D. 86 Raynaud, F. 32 R\$bas,E. 206 Reiter.RJ. 325 Rigual,R. 245 Ruan,D.Y. 98

Schengrund, C.-L. 1 Sewerynek, E. 325 Shen,E. 117 ShengJ.Z. 174 Shirahata, M. 298 Shiu,S.Y.W. 212

Skowronska-Jozwiak, E. 72 Stensaas, L.J. 109

Sun,S.-Y. 124

Tai,K.K. 174 Tam, S.M. 19 Tang, H. 117 Tang, J. 338 Tang,P.L. 311 Tian, Q. 338

Vicario, I. 245

Wan,Q. 24 Wang,M. 225 Wang,Z.-Z. 109 Warashina, A. 195 Wei,YJ. 338 Wong,N.S. 174 Wong,T.M. 133,174

Xu,F. 225 Xu,K. 150

Yang,C.C.H. 59

Zhu,D.-N. 124 
